\title{
Periodic permanent waves in an anharmonic chain with nearest-neighbour interaction
}

\author{
T P Valkering \\ Department of Applied Physics, Twente University of Technology, PO Box 217. \\ Enschede, The Netherlands
}

Received 2 February 1978 , in final form 7 April 1978

\begin{abstract}
The existence of longitudinal periodic permanent waves in a one-dimensional translationally invariant anharmonic chain with nearest-neighbour interaction is established by means of variational methods. A general expression for the energy is given in terms of the dispersion relation. The interaction potential is not specified in detail. Fundamental assumptions are: the potential has a vertical asymptote for small distance and a horizontal one for large distance between two neighbouring particles. In the limit for high energy the wave looks like an infinite series of 'spikes' with finite amplitude and 'infinite' velocity.
\end{abstract}

\section{Introduction}

Consider a one-dimensional infinite chain of unit masses. We assume nearest-neighbour interaction with a potential $V\left(r_{n}-r_{n-1}\right)$, where $r_{n}$ is the coordinate of the $n$th particle with respect to its equilibrium position.

Periodic permanent wave solutions are known for particular choices for $V$ (Toda 1975, Leo et al 1977). In the present paper we discuss the existence of these permanent waves for a general potential of the type as shown in figure 1. Essential features are the vertical and the horizontal asymptote and the inflection point at $r=\Delta_{0}$. In particular we are interested in the consequences of the asymptotes for the wave and for its dispersion relation. This type of potential is typical for longitudinal waves. Transversal waves should be described with an even and bounded potential.

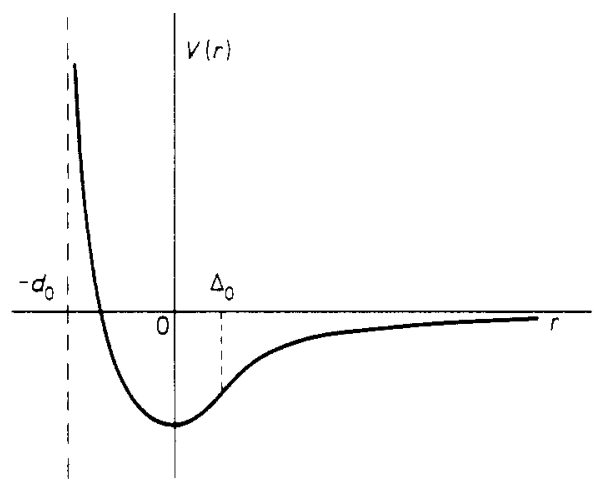

Figure 1. Typical interaction potential. 
In particular the potential is assumed to have the following properties:

(i) $V(r)$ is at least once continuously differentiable $\left(r>-d_{0}\right)$;

(ii) the derivative $V^{\prime}(r)$ is monotonically increasing (decreasing) if $-d_{0}<r<$ $\Delta_{0}\left(r>\Delta_{0}\right)$

(iii) $V(r)$ is three times continuously differentiable in a neighbourhood of $r=\Delta_{0}$ and $V^{\prime \prime \prime}\left(\Delta_{0}\right) \neq 0$.

We shall outline now the essentials of the methods and results. To describe a periodic permanent wave we shall introduce a function $s(\theta)$ with period $2 \pi$, such that $\omega s(\omega t-n k)$ gives the velocity of the $n$th particle in the chain. The constants $\omega$ and $k$ are respectively the circular frequency and the wavenumber. The function $s$ will be considered as element of a real Hilbert space of square integrable functions. The inner product being given by

$$
\langle s, v\rangle=\frac{1}{2 \pi} \int_{-\pi}^{\pi} v(\theta) s(\theta) \mathrm{d} \theta,
$$

the time average of the kinetic energy per particle in the wave equals

$$
\frac{1}{2} \omega^{2}\langle s, s\rangle .
$$

Further we define in terms of the potential $V$ for a given wavenumber $k$ a functional $\mathscr{V}(s)$ on a domain in $\mathscr{H}$, such that $\mathscr{V}(s)$ equals the time average of the potential energy per particle. The equation of motion for $s$ turns out to have the form

$$
\omega^{2} \operatorname{grad}\langle s, s\rangle=\operatorname{grad} \mathscr{V}(s),
$$

i.e. a solution realises an extremum of the potential energy for a constant kinetic energy.

Due to the singularity in $V$ it is not possible to define $\mathscr{V}$ on all of $\mathscr{H}$. There exists, however, a positive number $R_{0}$ such that $\mathscr{V}$ is defined on the open ball in $\mathscr{H}$ around the origin with radius $R_{0}$. By means of standard theorems it will be shown that for any given real parameter $R$ in the range $0 \leqslant R<R_{0}$ there exists at least one solution pair $\left\{\omega^{2}(R), s(R)\right\}$ with $\langle s, s\rangle=R^{2}$. Note that both $\omega^{2}$ and $s$ depend on the a priori given wavenumber $k$.

This solution pair will be investigated in more detail for the case that $R$ has a value close to $R_{0}$. This investigation is based upon the fact that there exists an element, say $s_{0}$, in $\mathscr{H}$ with norm $R_{0}$ such that $\mathscr{V}$ is singular in $s_{0}$, i.e. $\mathscr{V}(s)$ is unbounded in any small surrounding of $s_{0}$. Because the solution of $(1.3)$ realises the maximum of $\mathscr{V}(s)$ on the sphere in $\mathscr{H}$ with radius $R$, this solution is close to $s_{0}$ if $R$ is close to $R_{0}$. The velocity of the wave, which equals $\omega / k$, tends to infinity if $R$ tends to $R_{0}$, and the same holds for the time average of the energy per particle. The solution $s$ in this case represents an infinite train of equidistant compressional pulses running through the chain with 'infinite' velocity. The distance between the pulses equals $2 \pi / k$ and their width equals approximately the equilibrium distance between two neighbouring particles.

\section{The equation for periodic permanent waves}

The equations of motion are

$$
\ddot{r}_{n}=-V^{\prime}\left(r_{n}-r_{n-1}\right)+V^{\prime}\left(r_{n+1}-r_{n}\right) .
$$


A periodic permanent wave can be written as

$$
r_{n}(t)=r(\omega t-n k)+n \Delta,
$$

where $r$ is a periodic function with zero average and $\Delta$ is a parameter that equals the expansion of the chain per particle. The period of $r$ being chosen as $2 \pi, \omega$ and $k$ are respectively the circular frequency and the wavenumber of the wave. Substitution of (2.2) into (2.1) gives, if $\omega t-n k$ is replaced by $\theta$,

$\omega^{2}\left(\frac{\mathrm{d}}{\mathrm{d} \theta}\right)^{2} r(\theta)=-V^{\prime}(r(\theta)-r(\theta+k)+\Delta)+V^{\prime}(-r(\theta)+r(\theta-k)+\Delta)$.

To give this equation a well suited form we introduce the linear transformation $B$ that transforms a period function $g(\theta)$ according to

$$
B g(\theta)=g\left(\theta-\frac{1}{2} k\right)-g\left(\theta+\frac{1}{2} k\right) \text {. }
$$

With the aid thereof we write $(2.3)$ as

$$
\omega^{2}\left(\frac{\mathrm{d}}{\mathrm{d} \theta}\right)^{2} r(\theta)=B V^{\prime}(B r(\theta)+\Delta)
$$

We proceed with the definition of a linear transformation $A$

$$
A g(\theta)=-\int_{\theta-\frac{1}{2} k}^{\theta+\frac{1}{2} k} g(\tau) \mathrm{d} \tau \text {. }
$$

One easily verifies

$$
A \frac{\mathrm{d}}{\mathrm{d} \theta}=\frac{\mathrm{d}}{\mathrm{d} \theta} A=B
$$

with the aid of which we obtain instead of $\left(2.3^{\prime}\right)$

$$
\frac{\mathrm{d}}{\mathrm{d} \theta}\left[\omega^{2} s(\theta)-A V^{\prime}(A s(\theta)+\Delta)\right]=0, \quad s(\theta)=\frac{\mathrm{d}}{\mathrm{d} \theta} r(\theta) .
$$

The procedure is now as follows: for a given value of $k$ and $\Delta$ one finds $\omega^{2}$ and $s$ such that (2.6) holds. Because of the periodicity of $r$ the function $s$ should have zero average and for the same reason we choose $k$ within the interval $[-\pi,+\pi]$. Because change of sign of $k$ corresponds with change of direction of the corresponding wave, we restrict $k$ to the range

$$
0<k \leqslant \pi \text {. }
$$

Obviously we choose $\Delta>-d_{0}$.

\section{Mathematical preliminaries}

Consider the set $\mathscr{H}$ of real functions defined on the real axis

$\mathscr{H}=\left\{f \mid f(\theta)=f(-\theta) ; f(\theta+2 \pi)=f(\theta) ; \int_{-\pi}^{\pi} f(\tau) \mathrm{d} \tau=0 ; \int_{-\pi}^{\pi} f^{2}(\tau) \mathrm{d} \tau<\infty\right\}$. 
With inner product

$$
\langle f, g\rangle=\frac{1}{2 \pi} \int_{-\pi}^{\pi} f(\tau) g(\tau) \mathrm{d} \tau
$$

we may view $\mathscr{H}$ as a Hilbert space with orthonormal basis

$$
\phi_{n}=\sqrt{2} \cos n \theta, \quad n=1,2, \ldots
$$

Now we turn to the linear transformation $A$ defined in (2.5). Define the element $h_{k} \in \mathscr{H}$

$$
h_{k}(\theta)= \begin{cases}-1+\frac{k}{2 \pi}, & -\frac{1}{2} k<\theta<+\frac{1}{2} k, \\ +\frac{k}{2 \pi}, & \text { elsewhere }\end{cases}
$$

with norm

$$
\left\|h_{k}\right\|^{2}=\frac{k}{2 \pi}\left(1-\frac{k}{2 \pi}\right)
$$

With (2.5) and with the third property in (3.1) one easily verifies

$$
A g(\theta)=\left\{\begin{array}{l}
\int_{-\pi}^{\pi} h_{k}(\tau+\theta) g(\tau) \mathrm{d} \tau, \quad g \in \mathscr{H}, \\
2 \pi\left\langle h_{k \theta}, g\right\rangle, \dagger
\end{array}\right.
$$

where $h_{k \theta}(\tau)=h_{k}(\tau+\theta)$.

The following two properties of $A$ are basic for this paper:

(i) $\mathrm{Ag}(\theta)$ is a continuous function of $\theta$ and

$$
\sup _{\theta}|A g(\theta)| \leqslant 2 \pi\left\|h_{k}\right\|\|g\|
$$

(ii) $A$ is a completely continuous and self-adjoint linear transformation that maps $\mathscr{H}$ into $\mathscr{H}$.

The first property follows with Schwarz' inequality and (3.6b). To prove the second, we first state that $A$ is a bounded linear transformation that maps $\mathscr{H}$ into $\mathscr{H}$, which follows from (3.6) and (i). Then we notice that any element $\phi_{n}$ of the basis (3.3) is eigenvector of $A$

$$
A \phi_{n}=-\frac{2}{n} \sin \frac{n k}{2} \phi_{n}
$$

The properties of the spectrum of $A$ then immediately imply (ii) (cf Helmberg 1969 , $\$ 28$ ).

\section{Existence of solutions}

First we write (2.6) in a form that is suited to apply variational methods, for which we refer to Vainberg (1964) (a well-suited reference is also Temme 1976, chapter X).

$\dagger$ The symbol (, ) denotes here the inner product in the space defined by (3.1) without the requirement that the functions are even. 
Define the positive number $R_{0}$

$$
R_{0}=\frac{d_{0}+\Delta}{2 \pi\left\|h_{k}\right\|}
$$

From (3.7) it then follows if $\|g\|<R_{0}$

$$
\inf _{\theta}(A g(\theta)+\Delta)>\Delta-\sup _{\theta}|A g(\theta)|>-d_{0}
$$

Now define the functional $\mathscr{V}_{\Delta}$ on the open ball $B_{R_{0}}$ in $\mathscr{H}$

$$
\vartheta_{\Delta}(s)=\frac{1}{2 \pi} \int_{-\pi}^{\pi} V(A s(\tau)+\Delta) \mathrm{d} \tau, \quad\|s\|<R_{0} .
$$

The domain of definition is chosen to be $B_{R_{0}}$ in order to guarantee that the argument of $V$ in the integrand is larger than $-d_{0}(\mathrm{cf}(4.2))$. For the gradient of $\mathscr{V}$ we have by definition (Vainberg 1964, \$5)

$$
\lim _{\epsilon \rightarrow 0} \frac{1}{\epsilon}\left|\mathscr{V}_{\Delta}(s+\epsilon h)-\mathscr{V}_{\Delta}(s)\right|=\left\langle\operatorname{grad} \mathscr{V}_{\Delta}(s), h\right\rangle
$$

One easily verifies (cf the third property in (3.1))

$$
\operatorname{grad} \mathscr{V}_{\Delta}(s)=A\left(V^{\prime}(A s+\Delta)-\frac{1}{2 \pi} \int_{-\pi}^{\pi} V^{\prime}(A s(\tau)+\Delta) \mathrm{d} \tau\right) .
$$

The second term on the right is necessary because the gradient should be an element of $\mathscr{H}$. As far as solutions $s \in \mathscr{H}$ are considered, equation (2.6) can now be written as

$$
\omega^{2} s=\operatorname{grad} \mathscr{V}_{\Delta}(s)
$$

The basic ingredients for the existence proof for solutions to this equation are given in the following lemmas.

Lemma 4.1. The functional $\mathscr{V}_{\Delta}$ is weakly continuous on any closed ball $B_{R}, R<R_{0}$.

Proof. From inequality (3.7) we infer that the set

$$
V^{\prime}(A s+\Delta)-\frac{1}{2 \pi} \int_{-\pi}^{\pi} V^{\prime}(A s(\tau)+\Delta) \mathrm{d} \tau, \quad\|s\|<R,
$$

is bounded. Compactness of $A$ then implies (cf (4.5)) that grad $\mathscr{V}_{\Delta}$ maps any bounded subset of $B_{R}$ into a compact set, and so grad $\mathscr{V}_{\Delta}$ is a compact operator by definition. A compact gradient corresponds with a weakly continuous functional (Vainberg 1964, theorem 8.2).

\section{Lemma 4.2}

(i) Any extremum of $\mathscr{V}_{\Delta}$ within the open ball $B_{R_{0}}$ is necessarily situated at $s=0$.

(ii) Zero is a minimum point if $\Delta<\Delta_{0}$ and it is a maximum point if $\Delta>\Delta_{0}$.

(iii) If $\Delta=\Delta_{0}$, the functional $\mathscr{V}_{\Delta}$ has no extremum within the ball $B_{R_{0}}$.

\section{Proof}

(i) If $\mathscr{V}_{\Delta}$ has an extremum in $B_{R_{0}}$, say at $s_{1}$, then it necessarily holds that grad $\mathscr{V}_{\Delta}\left(s_{1}\right)=0$ (Vainberg 1973, theorem 9.1.1), which implies (cf (4.5)) $s_{1}=0$. 
(ii) Below we shall show that $\mathscr{V}_{\Delta}$ is convex in a neighbourhood of $s=0$ if $\Delta<\Delta_{0}$, or equivalently that grad $\mathscr{V}_{\Delta}$ is monotone. The proof then follows from Vainberg (1973, theorem 9.1.2). If $\Delta>\Delta_{0}$ the same argument can be applied on the functional $-\mathscr{V}_{\Delta}$. To show that grad $\mathscr{V}_{\Delta}$ is monotone we write (cf (4.5))

$\left\langle\operatorname{grad} \mathscr{V}_{\Delta}\left(s_{1}\right)-\operatorname{grad} \mathscr{V}_{\Delta}\left(s_{2}\right), s_{1}-s_{2}\right\rangle$

$$
=\frac{1}{2 \pi} \int_{-\pi}^{\pi}\left[V^{\prime}\left(A s_{1}(\theta)+\Delta\right)-V^{\prime}\left(A s_{2}(\theta)+\Delta\right)\right]\left[A s_{1}(\theta)-A s_{2}(\theta)\right] \mathrm{d} \theta \text {. }
$$

By assumption ( $\$ 1$; property (ii)) there is for any $\Delta<\Delta_{0}$ a $\delta>0$ such that $V(x)$ is monotonically increasing if $\Delta-\delta<x<\Delta+\delta$. Because of (3.7) there is for any $\delta>0$ an $\epsilon>0$ such that

$$
\sup |A s(\theta)|<\delta \quad \text { if } \quad\|s\|<\epsilon .
$$

Consequently both factors in the integrand in (4.8) have the same sign for any $-\pi \leqslant \theta \leqslant \pi$ if $\left\|s_{1,2}\right\|<\epsilon$ so that the integrand is non-negative. By definition it follows that grad $\mathscr{V}_{\Delta}$ is (in fact strictly) monotone in $B_{\epsilon}$.

(iii) If we write down the Taylor series of $\mathscr{V}_{\Delta_{0}}(x h)$ around $x=0$ ( $h$ is a fixed element in $\mathscr{H}$ ) then we see immediately that the statement follows from property (iii) in $\$ 1$.

\section{Lemma 4.3}

(i) If $\Delta<\Delta_{0}\left(\Delta>\Delta_{0}\right)$ the functional $\mathscr{V}_{\Delta}$ achieves its supremum (infimum) in the closed ball $B_{R}, R<R_{0}$, on the boundary $S_{R}$.

(ii) If $\Delta=\Delta_{0}$ the functional $\mathscr{V}_{\Delta}$ achieves both its infimum and its supremum on $S_{R}$.

Proof. Because $\mathscr{H}$ is a Hilbert space, the closed ball $B_{R}$ is both weakly closed and weakly compact. Further $\mathscr{V}_{\Delta}$ is weakly continuous (lemma 4.1). Consequently the generalised theorem of Weierstrass applies (Vainberg 1964, theorem 13.2). It follows that $\mathscr{V}_{\Delta}$ achieves both its infimum and its supremum on the closed ball $B_{R}$. Application of lemma 4.2 completes the proof.

Now the main result of the present paper can be formulated and proved.

Theorem 4.4. For any $\Delta<\Delta_{0}\left(\Delta>\Delta_{0}\right)$ and any positive $R<R_{0}$, equation (4.6) has a solution pair $\left\{\omega^{2}, \bar{s}\right\}$ with $\|\bar{s}\|=R$ and $\omega^{2}>0\left(\omega^{2}<0\right)$. The functional $\mathscr{V}_{\Delta}$ achieves its supremum on the closed ball $B_{R}$ in $\bar{s}$.

Proof. First consider the case $\Delta<\Delta_{0}$. Let $\bar{s}$ denote an element on $S_{R}$ at which $\mathscr{V}_{\Delta}$ achieves its supremum according to lemma 4.3(i). According to Lusternik's theorem (Vainberg 1964, theorems $12.1,2$ ), there is a real number $\mu$, such that (4.6) holds in that extremal point with $\omega^{2}=\mu$. This number is surely non-zero, because $\mu=0$ implies $\operatorname{grad} \mathscr{V}_{\Delta}(\bar{s})=0$ and so $\bar{s}=0$, which contradicts $\|\bar{s}\|=R$. Further we have $\mathscr{V}_{\Delta}(\bar{s})>\mathscr{V}_{\Delta}\left(s^{\prime}\right)$ where $s^{\prime}$ is any interior point of $B_{R}$. Assume $\mu<0$. Then grad $\mathscr{V}_{\Delta}(\bar{s})$ is directed inwards, and so there is an interior point of $B_{R}$, say $s^{\prime \prime}$, with $\mathscr{V}_{\Delta}\left(s^{\prime \prime}\right)>\mathscr{V}_{\Delta}(\bar{s})$. This contradicts the first inequality. We conclude to $\mu>0$. If $\Delta>\Delta_{0}$ the same type of argument applies. 
Note that as a consequence of the inequality

$$
\Delta+\sup _{\theta}|A s(\theta)|<\Delta+2 \pi\left\|h_{k}\right\| R_{0}=d_{0}+2 \Delta, \quad\|s\|<R_{0},
$$

the values of the potential $V(r)$ for $r>d_{0}+2 \Delta$ do not influence either the functional $\mathscr{V}_{\Delta}$ or the solutions of (4.6).

The solution to (4.6) guaranteed by the above theorem is not unique. Although one might expect that for almost all values of $R$ there is only one $s$ on $S_{R}$ for which $\mathscr{V}_{\Delta}$ has an absolute maximum, this is not the only solution to (4.6). Any $s$ for which $\mathscr{V}_{\Delta}$ is stationary with respect to the surface $S_{R}$ satisfies.

Let us consider as an example the case of a harmonic chain: $V(r)=\frac{1}{2} b r^{2}$. Equation (4.6) then reads $\omega^{2} s=b A^{2} s$. This equation has an infinity of solution pairs (cf (3.8)):

$$
\left\{\omega_{i}^{2}(R), s_{i}(R)\right\}=\left\{b 4 i^{-2} \sin ^{2} \frac{1}{2} i k, R_{i} \sqrt{2} \cos i \theta\right\} .
$$

One easily verifies that the maximum of $\mathscr{V}_{\Delta}(s)$, which is given by

$$
\mathscr{V}_{\Delta}(s)=\frac{b}{4 \pi} \int_{-\pi}^{\pi}(A s(\tau)+\Delta)^{2} \mathrm{~d} \tau,
$$

is realised by the solution with $i=1$. In the present anharmonic case we expect the analogous situation, i.e. the existence of a set of solutions $\left\{\omega_{i}^{2}\left(R_{i}\right), s_{i}\left(R_{i}\right)\right\}$. For small values of the parameters $R_{i}$ these solutions should tend to the harmonic solutions. A detailed study of the complete set of solutions to (4.6), however, is beyond the scope of this paper.

In the case that $\Delta>\Delta_{0}, \omega^{2}$ is negative so that $\omega$ is imaginary. This indicates unstable behaviour, which can be seen in the following way. The parameter $\Delta$ gives the expansion of the chain per particle. We can incorporate this in the equations of motion ( 2.1 ) by introducing a new interaction potential

$$
\tilde{V}\left(\tilde{r}_{n}-\tilde{r}_{n-1}\right)=V\left(\tilde{r}_{n}-\tilde{r}_{n-1}+\Delta\right)-V^{\prime}(\Delta)\left(\tilde{r}_{n}-\tilde{r}_{n-1}\right),
$$

where $\tilde{r}_{n}$ is the coordinate of the $n$th particle with respect to the new equilibrium position: it holds that $\tilde{r}_{n}=r_{n}-n \Delta$. The linear term in the potential is added to guarantee that $\tilde{V}$ has an extremum if $\tilde{r}_{n}-\tilde{r}_{n-1}=0$. One easily sees that this extremum is a minimum if $\Delta<\Delta_{0}$. It is a maximum, however, if $\Delta>\Delta_{0}$. This implies that the zero solution of the equations of motion for the stretched chain

$$
\tilde{r}_{n}=-\tilde{V}^{\prime}\left(\tilde{r}_{n}-\tilde{r}_{n-1}\right)+\tilde{V}^{\prime}\left(\tilde{r}_{n+1}-\tilde{r}_{n}\right)
$$

is unstable if $\Delta>\Delta_{0}$. From now on we restrict the range of $\Delta$ by

$$
-d_{0}<\Delta<\Delta_{0}
$$

\section{The energy}

With the aid of an assumption about the continuity of a solution $\omega^{2}(R), s(R)$ with respect to $R$, the energy of the wave will be written in terms of the square of the frequency: $\omega^{2}(R)$. The motion of the $n$th particle of the chain is described by

$$
\frac{\mathrm{d}}{\mathrm{d} t} r_{n}(t)=\frac{\mathrm{d}}{\mathrm{d} t} r(\omega t-n k)=\omega s(\omega t-n k),
$$




$$
\begin{aligned}
r_{n}(t)-r_{n-1}(t) & \\
= & r(\omega t-n k)-r(\omega t-n k+k)+\Delta \\
= & (B r)\left(\omega t-n k+\frac{1}{2} k\right)+\Delta=(A s)\left(\omega t-n k+\frac{1}{2} k\right)+\Delta .
\end{aligned}
$$

For the time average of the kinetic and the potential energy per particle we then have respectively for any solution of $(4.6)$

$$
\begin{gathered}
\bar{T}=\frac{\omega}{2 \pi} \int_{-\pi / \omega}^{\pi / \omega} \frac{1}{2}\left(\frac{\mathrm{d}}{\mathrm{d} t} r(t)\right)^{2} \mathrm{~d} t=\frac{\omega^{2}}{2} \frac{1}{2 \pi} \int_{-\pi}^{\pi} s^{2}(\tau) \mathrm{d} \tau, \\
\bar{V}=\frac{\omega}{2 \pi} \int_{-\pi / \omega}^{\pi / \omega} V\left(r_{n}(\tau)-r_{n-1}(\tau)+\Delta\right) \mathrm{d} \tau=\frac{1}{2 \pi} \int_{-\pi}^{\pi} V(A s(\tau)+\Delta) \mathrm{d} \tau=\mathscr{V}_{\Delta}(s) .
\end{gathered}
$$

For any arbitrary $s$, the functional $\mathscr{V}_{\Delta}(s)$ is given by the expression (cf Vainberg 1964 , $\$ 2)$

$$
\mathscr{V}_{\Delta}(s)=\mathscr{V}_{\Delta}(0)+\int_{L}\left\langle\operatorname{grad} \mathscr{V}_{\Delta}(\tilde{s}(\alpha)), \mathrm{d} \tilde{s}(\alpha)\right\rangle,
$$

where the path of integration $L$ connects 0 and $s$ and is represented by a suitable abstract function $\tilde{s}(\alpha)$. For (5.4) to be valid it is sufficient that $\tilde{s}(\alpha)$ is a continuous function of $\alpha$ and that it is of bounded variation. Now assume that the solution $\bar{s}(R)$ given in theorem 4.4 satisfies these properties. Making use of (4.6) we then find

$$
\mathscr{V}_{\Delta}\left(f f^{-} s(R)\right)=\mathscr{V}_{\Delta}(0)+\int_{0}^{R} \omega^{2}\left(R^{\prime}\right)\left\langle\bar{s}\left(R^{\prime}\right), \mathrm{d} \bar{s}\left(R^{\prime}\right)\right\rangle .
$$

Finally we conclude with (5.3) that the total energy of the solution given in theorem 4.4 is given by

$$
\bar{T}(R)+\bar{V}(R)=\frac{1}{2} \omega^{2}(R) R^{2}+\int_{0}^{R} \omega^{2}\left(R^{\prime}\right) R^{\prime} \mathrm{d} R^{\prime}+V(\Delta) .
$$

The validity of (5.6) depends on the assumption concerning $\vec{s}(R)$. It remains valid if there are discontinuities. Consider the case of one discontinuity, say at $R^{\prime \prime}$. Then we have the equalities

$$
\begin{aligned}
& -\mathscr{V}_{\Delta}(0)+\lim _{R^{\prime} \uparrow R^{\prime \prime}} \mathscr{V}_{\Delta}\left(\bar{s}\left(R^{\prime}\right)\right)=\int_{0}^{R^{\prime \prime}} \omega^{2}\left(R^{\prime}\right) R^{\prime} \mathrm{d} R^{\prime}, \\
& -\lim _{R^{\prime} \downarrow R^{\prime \prime}} \mathscr{V}_{\Delta}\left(\bar{s}\left(R^{\prime}\right)\right)+\mathscr{V}_{\Delta}(\bar{s}(R))=\int_{R^{\prime \prime}}^{R} \omega^{2}\left(R^{\prime}\right) R^{\prime} \mathrm{d} R^{\prime} .
\end{aligned}
$$

If we add both equations, the limits cancel because they both equal the maximal value of $\mathscr{V}_{\Delta}$ on the spheres $S_{R^{\prime \prime}}$, and again we arrive at (5.5).

\section{The solution with high energy}

In this section we shall discuss the shape of the solution and bounds for the kinetic and the potential energy per particle if $R$ tends to $R_{0}$. The functional $\mathscr{V}$ is defined on any ball in $\mathscr{H}$ with radius (cf $\S 4) R<R_{0}$. On the sphere $S_{R_{0}} \mathscr{V}_{\Delta}$ is defined everywhere where the inequality in (3.7) holds. From $(3.6 b)$ it follows that the equality is satisfied 
only if $g=c_{0} h_{k}$ or $g=c_{0} h_{k \pi}$, where $c_{0}$ is a constant, so we conclude that $\mathscr{V}_{\Delta}$ is defined on $S_{R_{0}}$ apart from singularities at

$$
s_{0(\pi)}=-h_{k(\pi)} \frac{1}{\left\|h_{k}\right\|} R_{0} .
$$

This fact forms the basis of the following theorem.

Theorem 6.1. Let the singularity of $V(r)$ at $r=-d_{0}$ be non-integrable, i.e.

$$
\int_{0}^{-d_{0}(1-\alpha)} V(r) \mathrm{d} r \rightarrow-\infty \quad \text { if } \quad \alpha \rightarrow 0 .
$$

Then for any $\epsilon>0$ there is a $\delta_{0}>0$ such that equation (4.6) for any positive $\delta \leqslant \delta_{0}$ has a solution satisfying

$$
\|s\|=R_{0}(1-\delta), \quad\left\|s-s_{0}\right\| \leqslant \epsilon R_{0}
$$

and

$$
\sup _{\theta}|A s| \leqslant\left(d_{0}+\Delta\right)(1-\delta), \quad \sup _{\theta}\left|A s-A s_{0}\right| \leqslant \epsilon\left(d_{0}+\Delta\right) .
$$

Proof. Choose $\epsilon>0$. There is a constant $M$ such that $\mathscr{V} \leqslant M$ for any $s$ satisfying

$$
\|s\| \leqslant R_{0}, \quad\left\|s-s_{0}\right\| \geqslant \epsilon R_{0} .
$$

From a straightforward calculation of $\mathscr{V}_{\Delta}\left((1-\delta) s_{0}\right)$ the existence of a $\delta_{0}<\epsilon$ such that $\mathscr{V}_{\Delta}\left((1-\delta) s_{0}\right)>M$ for $0<\delta<\delta_{0}$ follows immediately. Consequently $\mathscr{V}$ assumes its extremum on $S_{R_{0}(1-\delta)}$ within the $\epsilon R_{0}$ neighbourhood of $s_{0}$. This proves (6.2). With the aid of (3.7) and (4.1), equation (6.3) follows immediately.

Equation (6.2) in this theorem shows that the velocity of any particle (cf (5.1)) during the wave motion is approximately (in the norm of $\mathscr{H}$ ) given by $\omega s_{0}$ if $R$ is close to $R_{0}$. The relative distance between the particles, however, is approximated uniformly in the argument $\theta$ by $A s_{0}+\Delta(\operatorname{cf}(5.2),(6.3))$. The latter result can be visualised (cf figure 2 ) in an easy way. To do this we define the functions (see figure 2) $g_{+,-}(\theta)$

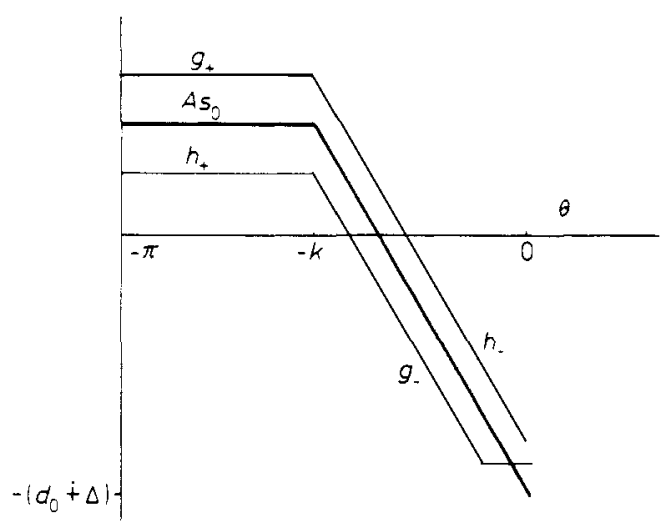

Figure 2. The functions $A s_{0}(\theta), h_{+,-}(\theta)$ and $g_{+,-}(\theta)$. 
and $h_{+,-}(\theta)$ as follows: they equal zero apart from

$$
\begin{array}{lll}
g_{+}(\theta)=A s_{0}(\theta)+\epsilon\left(d_{0}+\Delta\right) & \text { if } & A s_{0}(\theta)+\epsilon\left(d_{0}+\Delta\right) \geqslant 0, \\
h_{+}(\theta)=A s_{0}(\theta)-\epsilon\left(d_{0}+\Delta\right) & \text { if } & A s_{0}(\theta)-\epsilon\left(d_{0}+\Delta\right) \geqslant 0, \\
g_{-}(\theta)=A s_{0}(\theta)-\epsilon\left(d_{0}+\Delta\right) & \text { if } & 0>A s_{0}(\theta)-\epsilon\left(d_{0}+\Delta\right) \geqslant-\left(d_{0}+\Delta\right)(1-\delta), \\
g_{-}(\theta)=-\left(d_{0}+\Delta\right)(1-\delta) & \text { if } & A s_{0}(\theta)-\epsilon\left(d_{0}+\Delta\right) \leqslant-\left(d_{0}+\Delta\right)(1-\delta), \\
h_{-}(\theta)=A s_{0}(\theta)+\epsilon\left(d_{0}+\Delta\right) & \text { if } & A s_{0}(\theta)+\epsilon\left(d_{0}+\Delta\right) \leqslant 0,
\end{array}
$$

where $A s_{0}$ is given by (cf (6.1) and (3.4))

$$
A s_{0}(\theta)= \begin{cases}\frac{R_{0}}{\left\|h_{k}\right\|} \frac{k^{2}}{2 \pi}, & -\pi \leqslant \theta \leqslant-k, \\ \frac{R_{0}}{\left\|h_{k}\right\|}\left(-\theta-k+\frac{k^{2}}{2 \pi}\right), & -k<\theta \leqslant 0 .\end{cases}
$$

One easily concludes that the actual value of $A s(\theta)$ is lying in the area enclosed by the non-zero parts of $h_{+,-}$and $g_{+,-}$in figure 2 . define

To prepare the ground for the calculation of bounds for the kinetic energy we

$$
I_{+,-}=\frac{1}{\pi} \int_{i_{+,-}} A s(\tau)\left[V^{\prime}(A s(\tau)+\Delta)-V^{\prime}(\Delta)\right] \mathrm{d} \tau,
$$

where $i_{+,-}$is the interval of $[-\pi, 0]$ where $A s(\theta)$ is positive or negative respectively. Taking the inner product of both sides of (4.6) and $s$ one finds, making use of the fact that $s$ has zero average,

$$
\omega^{2} R^{2}=I_{+}+I_{-} .
$$

Careful inspection of figure 2 leads to the following bounds for $I_{-}$:

$\frac{1}{\pi} \int_{-\pi}^{0} h_{-}(\tau)\left[V^{\prime}\left(h_{-}(\tau)+\Delta\right)-V^{\prime}(\Delta)\right] \mathrm{d} \tau \leqslant I_{-} \leqslant \frac{1}{\pi} \int_{-\pi}^{0} g_{-}(\tau)\left[V^{\prime}\left(g_{-}(\tau)+\Delta\right)-V^{\prime}(\Delta)\right] \mathrm{d} \tau$.

Calculation of these bounds is straightforward. The results are

$$
\begin{aligned}
I_{-} \leqslant \frac{k}{\pi}\left(1-\frac{k}{2 \pi}\right)\left(d_{0}+\Delta\right)\left(\left.(\epsilon+\delta)(1-\delta) V^{\prime}(\alpha+\Delta)\right|_{\alpha=-\left(d_{0}+\Delta\right)(1-\delta)} ^{\alpha=0}\right. \\
\left.+\frac{1}{2}(1-\delta)^{2} V^{\prime}(\Delta)+\frac{S_{\delta}}{\left(d_{0}+\Delta\right)^{2}}\right), \\
I_{-} \geqslant \frac{k}{\pi}\left(1-\frac{k}{2 \pi}\right)\left(d_{0}+\Delta\right)\left(\frac{1}{2}(1-\epsilon)^{2} V^{\prime}(\Delta)+\frac{S_{\epsilon}}{\left(d_{0}+\Delta\right)^{2}}\right),
\end{aligned}
$$

where $S_{\alpha}$ represents the surface of the shaded area in figure 3. In an analogous way bounds can be derived for $I_{+}$. These, however, are bounded for small $\epsilon$ and $\delta$, and their detailed form is less relevant.

We can formulate now the following theorem: 


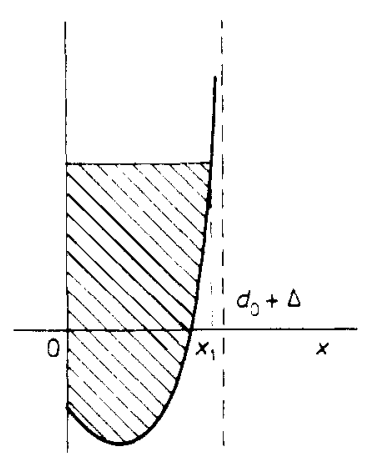

Figure 3. $V(-x+\Delta)$ against $x, x_{1}=(1-\alpha)\left(d_{0}+\Delta\right)$.

Theorem 6.2. Let the singularity of $V(r)$ at $r=-d_{0}$ be of degree $m>1$, i.e.

$$
V(r)=\mathrm{O}\left(r+d_{0}\right)^{-m} \quad \text { if } \quad r \downarrow-d_{0},
$$

then there exists for any $\epsilon>0$ a $\delta_{0}>0$ and there exist positive constants $M_{i}(k, \Delta)$ such that for the time average of the kinetic energy per particle of the wave corresponding with a solution given in theorem 6.1 , it holds that

$$
M_{1}+M_{2} \epsilon^{-m}<\bar{T}\left(R_{0}(1-\delta)\right)<M_{3}+M_{4} \epsilon \delta^{-m-1}+M_{5} \delta^{-m}, \quad 0<\delta<\delta_{0}
$$

Further there exist constants $N_{i}(k, \Delta)$ such that for the time average of potential energy it holds that

$N_{1}+N_{2} \epsilon^{-m+1}<\bar{V}\left(R_{0}(1-\delta)\right)<N_{3}+N_{4} \epsilon \delta^{-m}+N_{5} \delta^{-m+1}, \quad 0<\delta<\delta_{0}$.

Proof. The first part of the theorem is an immediate consequence of the bounds discussed above for $I_{+}$and $I_{-}$and of

$$
\bar{T}(R)=\frac{1}{2} \omega^{2} R^{2}=\frac{1}{2}\left(I_{+}+I_{-}\right)
$$

The second part follows from the same type of argument. Define

$$
H_{+,-}=\frac{1}{\pi} \int_{i_{+,-}} V(A s(\theta)+\Delta) \mathrm{d} \theta,
$$

where $i_{+,-}$is the interval of $[-\pi, 0]$ where $A s(\theta)+\Delta$ is positive or negative respectively. $H_{+}$is bounded for small $\epsilon$ and $\delta$. For $H_{-}$the same type of bounds can be formulated as was done for $I_{-}$in (6.10). The essential difference is that the singularity in the integrands in (6.10) is of degree $m+1$ because of the occurrence of $V^{\prime}$, whereas the singularity in the integrands occurring in the bounds for $H_{-}$is of degree $m$. This leads immediately to (6.14).

We conclude with a few remarks.

(i) Because $\epsilon$ can be chosen arbitrarily small and because of the condition $m>1$ in the theorem, the lower bounds in $(6.13,6.14)$ show that both the kinetic and the potential energy per particle are unbounded for $R$ in the neighbourhood of $R_{0}$. 
(ii) From (6.13) one derives the same type of bounds for the square of the phase velocity

$$
c^{2}=\omega^{2} / k^{2}=2 \bar{T}(R) /\left(R^{2} k^{2}\right) .
$$

Consequently the velocity of the wave is also unbounded if $R$ is in the neighbourhood of $R_{0}$.

(iii) If the singularity of $\bar{T}$, and so of $\omega^{2}$, is of degree $\alpha$ : i.e. if $\bar{T}$ is approximated by a constant times $\left(R-R_{0}\right)^{-\alpha}$, then the upper bound in (6.13) shows that $\alpha \leqslant m+1$. If one knows how $\delta_{0}$ depends on $\epsilon$, one can say more about $\alpha$.

(iv) The parameter $R$ does not have an immediate physical meaning. Note, however, that the potential energy per particle $V(R)$ varies from $V(\Delta)$ to infini if $R$ varies from zero to $R_{0}$. In fact, $\bar{V}(R)=\mathscr{V}_{\Delta}(\bar{s}(R))$ is a monotonically increasing, unbounded and continuous function of $R$. This follows from the continuity of the functional $\mathscr{V}_{\Delta}(s)$ and from the second statement of theorem 4.4. Consequently there is a one-to-one correspondence between $R$ in the range $\left[0, R_{0}[\right.$ and $\bar{V}$ in the range $[V(\Delta), \infty[$.

\section{Concluding remarks}

To conclude we apply the above results to a finite chain of $N$ particles. We describe the motion of this system with the aid of the infinite chain with periodic boundary conditions. In this way the system is interpreted as a closed circular chain of $N$ particles.

Now consider a solution of (4.6) with $k=2 \pi / N$. Let us assume that the distance between two neighbouring particles in the chain equals unity if the particles are at rest and if $\Delta=0$. The actual distance between two neighbouring particles is then given by

$$
\rho_{n}(t)=1+r_{n}(t)-r_{n-1}(t)=(A s)\left(\omega t-n k+\frac{1}{2} k\right)+1+\Delta .
$$

Obviously this quantity is periodic in $n$ with period $N$. If the parameter $R$ is close to $R_{0}$, the function $A s(\theta)$ is close to $A s_{0}(\theta)$ for all values of the argument $\theta$. Making use of (6.7) we then find at a particular time $t=\frac{1}{2} k / \omega$ approximately

$$
\rho_{n}= \begin{cases}1-d_{0}, & n=1, \\ \frac{d_{0}+\Delta}{N-1}+1+\Delta, & n=2, \ldots, N .\end{cases}
$$

This represents a sharp compressional pulse. This pulse travels through the closed chain of $N$ particles with velocity $c(R)=\omega(R) / k$, which tends to infinity if $R$ tends to $R_{0}$ (cf theorem 6.2 and following).

In this way one easily sees that a solution of (4.6) with $k=i(2 \pi / N)$ represents a set of $i$ equidistant pulses running with constant velocity through the closed circular chain of $N$ particles. The $N$ solutions for $k= \pm i(2 \pi / N), i=1, \ldots, N / 2$ represent the non-linear analogues of the normal modes for the linear chain.

We should realise, however, that stability for small perturbations is a necessary condition for such pulses to exist in a real physical system. This property, however, should be proved. Another important question is the existence of a solution representing two or more pulses with different velocity. If the pulses still exist after 
interaction, they may be calleci solitons. Both properties are essential for the physical interpretation of the solutions found in this paper and they are the subject of further study.

\section{References}

Helmberg G 1969 Introduction to Spectral Theory in Hilbert Spaces (Amsterdam: North-Holland)

Leo M, Leo R A and Soliani G 1977 Phys. Lett. 60A pp 283-6

Temme N M (ed) 1976 Nonlinear Analysis vol 2 (Amsterdam: Mathematisch Centrum Syllabus 26.2)

Toda M 1975 Phys. Rep. 18 1-124

Vainberg M M 1964 Variational Methods for the Study of Nonlinear Operators (San Francisco: HoldenDay)

1973 Variational Method and Method of Monotone Operators in the Theory of Nonlinear Equations (New York: Wiley) 